\title{
Eosinophil cationic protein and immunoglobulin levels in bron- choalveolar lavage fluid obtained from patients with chronic eosinophilic pneumonia
}

\author{
K.A. Boomars*, H. van Velzen-Blad**, P.G.H. Mulder, L. Koenderman++, \\ J-W.J. Lammers ${ }^{++}$, J.M.M. van den Bosch*
}

Eosinophil cationic protein and immunoglobulin levels in bronchoalveolar lavage fluid obtained from patients with chronic eosinophilic pneumonia. K.A. Boomars, H. van Velzen-Blad, P.G.H. Mulder, L. Koenderman, J-W.J. Lammers, J.M.M. van den Bosch. (C) ERS Journals Ltd 1996.

ABSTRACT: In chronic eosinophilic pneumonia (CEP), histopathological evidence exists for the degranulation of eosinophils and the release of various toxic proteins. In vitro studies have demonstrated the degranulation of eosinophils in response to aggregated and complexed immunoglobulins. The aims of this study were to investigate: 1) whether the eosinophil cationic protein (ECP) and immunoglobulin (Ig) levels in bronchoalveolar lavage (BAL) fluid from patients with CEP are increased compared to those of healthy controls; 2) and whether a relationship is present between immunoglobulin levels and ECP levels in BAL fluid from patients with CEP.

The BAL from 12 patients with CEP was selected, retrospectively, from all BAL analyses performed in our centre between 1986 and 1992. ECP levels were measured using a radioimmunoassay in BAL fluid of patients with CEP and 10 healthy controls.

ECP levels and immunoglobulin levels in BAL fluid from patients with CEP were found to be elevated compared to controls $(p<0.001)$. A relationship was found between IgA levels and ECP levels in BAL fluid from patients with CEP ( $r=0.72$; $\mathrm{p}=\mathbf{0 . 0 4 3}$ ).

In conclusion, eosinophil cationic protein and immunoglobulin levels were found to be increased in bronchoalveolar lavage fluid from patients with chronic eosinophilic pneumonia. The relationship found between immunoglobulin A levels and eosinophil cationic protein levels may suggest that immunoglobulin A could be involved in the degranulation of eosinophils in chronic eosinophilic pneumonia. Eur Respir J., 1996, 9, 2488-2493.
Depts of *Pulmonary Diseases and **Immunology, St. Antonius Hospital, Nieuwegein, The Netherlands. +Dept of Epidemiology and Biostatistics, Erasmus University, Rotterdam, The Netherlands. ${ }^{++}$Dept of Pulmonary Diseases, University Hospital, Utrecht, The Netherlands.

Correspondence: J.M.M. van den Bosch Dept of Pulmonary Diseases

St. Antonius Hospital

P.O. Box 2500

3450 EM Nieuwegein

The Netherlands

Keywords: Bronchoalveolar lavage chronic eosinophilic pneumonia eosinophil cationic protein immunoglobulins

Received: September 191995 Accepted after revision July 91996

This study was supported by a grant of Glaxo, BV, The Netherlands.
Chronic eosinophilic pneumonia (CEP) is an interstitial lung disease characterized by prolonged pulmonary infiltration associated (usually) with peripheral blood eosinophilia. The clinical presentation of the disease is variable and the onset (sub)acute [1-3]. The most common symptoms include fever, cough, malaise, night sweats and dyspnoea. Several studies have described patients who suffered from asthma before the onset of CEP, often in combination with an atopic history $[1,2$, $4,5]$.

Histopathological studies have demonstrated interstitial and alveolar infiltration consisting mainly of eosinophils and macrophages, small to moderate numbers of lymphocytes, and occasionally plasma cells $[6,7]$. The alveolar walls are usually thickened, and sometimes focal areas of fibrosis are present. The airspaces contain macrophages, which may be multinucleated and contain eosinophilic granules and Charcot-Leyden crystals. Moreover, numerous lysed eosinophils are observed as well as accumulation of free extracellular eosinophilic granules, eosinophil cationic protein (ECP) and major basic protein (MBP) [7]. Both granule proteins have cytotoxic effects $[8,9]$. The processes and stimuli which lead to the degranulation of eosinophils in CEP, remain to be elucidated. In vitro studies have shown that aggregated immunoglobulin A ( $\operatorname{Ig} \mathrm{A})$ (especially secretory $\operatorname{IgA}$ ) and aggregated immunoglobulin $\mathrm{G}$ (IgG) induce degranulation of eosinophils [10]. Other investigators have demonstrated that challenge of eosinophils (isolated from peripheral blood of patients with eosinophilia) with anti-human IgA and IgG monoclonal antibodies causes degranulation of eosinophils and secretion of ECP, whereas in response to anti-human $\operatorname{IgE}$ monoclonal antibodies only eosinophil peroxidase (EPO) was released $[11,12]$.

In the bronchoalveolar lavage (BAL) fluid from patients with various interstitial lung diseases, such as systemic sclerosis and idiopathic pulmonary fibrosis, and in adult respiratory distress syndrome, in which eosinophils are assumed to play a role, ECP levels were demonstrated to be elevated [13-15]. In BAL fluid from patients with CEP, ECP levels were found to be increased in three patients in a study by JANIN et al. [16], and in six patients in a recent study by SHIJUBo et al. [17]. 
The aims of this study were to investigate: 1) whether the ECP levels and the immunoglobulin levels in BAL fluid from patients with CEP are increased compared to ECP levels and immunoglobulin levels in BAL fluid from healthy controls; and 2) whether a relationship exists between immunoglobulin levels and ECP levels in BAL fluid from patients with CEP.

\section{Methods}

\section{Patients and controls}

The BAL of 12 patients with CEP was selected, retrospectively, from all BAL analyses performed in our centre between 1986 and 1994. The diagnosis of CEP was based on compatible clinical information, chest radiography, percentage of eosinophils and total eosinophil count in peripheral blood, and the BAL cell profile. There was no evidence of parasitic, fungal or bacterial infection. Patients were not suffering from drug-induced pneumonitis, extrinsic allergic alveolitis, collagen-vascular disease or any malignancy. Additionally, pulmonary function tests (vital capacity (VC), transfer factor for carbon monoxide $(T \mathrm{~L}, \mathrm{CO})$ and forced expiratory volume in one second (FEV1)) were performed.

Diagnosis of asthma was based on the criteria defined by the American Thoracic Society (ATS) [18]. All patients were initially treated with high doses of corticosteroids for 6-12 weeks; the maintenance dose depended on the clinical course. Eight out of 12 patients relapsed one or more times after the corticosteroid treatment was ended, or while they were still receiving low-dose corticosteroid therapy. The follow-up ended in January 1996. The follow-up period ranged 1.9-9.8 yrs (mean $6.8 \mathrm{yrs})$.

To compare the results of the cell counts and the immunoglobulin levels in BAL fluid of patients with CEP, we used the results of BAL fluid analysis of a historical control group consisting of 15 healthy subjects (all nonsmokers, Control Group A) [19]. In 8 out of 12 patients and 10 out of 15 controls, immunoglobulin levels had been determined. Supernatant from these control subjects was no longer available, therefore we were unable to use their BAL fluid for determination of ECP levels. For this reason, BAL fluid was obtained from a second control group (Group B), consisting of 10 control subjects. Both control groups consisted of healthy volunteers without any chest disease or chest abnormalities. The characteristics of the patients studied are presented in table 1. This study was approved by the Ethics Committee of our hospital.

\section{Bronchoalveolar lavage}

BAL was performed during fibreoptic bronchoscopy as reported previously [20]. At the same time, blood samples were taken. In brief, the procedure was as follows. After premedication with atropine, and sometimes diazepam or codeine, and local anaesthesia of the larynx and bronchial tree with $0.5 \%$ tetracaine, BAL was performed by standardized washing of the middle lobe with four $50 \mathrm{~mL}$ aliquots of sterile saline solution $(0.9 \%$ $\mathrm{NaCl}$ ) at room temperature. Lavage fluid samples, kept on ice in a siliconized specimen trap, were centrifuged ( $350 \times \mathrm{g}$ for $10 \mathrm{~min}$ ) immediately after the lavage procedure and separated into cells and supernatant. The cells of the last three aliquots were pooled, washed twice, counted and suspended in minimal essential medium (Gibco, Grand Island, New York, USA) supplemented with $1 \%$ bovine serum albumin (Organon, Teknika, Boxtel, The Netherlands). Preparations of the cell suspension were made in a cytocentrifuge (Shandon). Cytospin slides of BAL cells were stained with May-Grünwald-Giemsa (Merck, Darmstadt, Germany) for cell differentiation counts. At least 1,000 cells were counted. Supernatants were stored frozen at $-70^{\circ} \mathrm{C}$.

Albumin concentrations in BAL fluid were determined turbidimetrically (by means of a Cobas Fara, Roche) with rabbit anti-human albumin antiserum (Dako, Glostrup, Denmark). Immunoglobulin M (IgM), IgG and $\operatorname{IgA}$ concentrations in BAL fluid were determined by enzymelinked immunosorbent assay (ELISA). Briefly, microtitre plates were coated with rabbit anti-human-isotype antiserum (anti-IgM, (CLB, Amsterdam, The Netherlands), anti-IgG and anti-IgA (Dako, Glostrup, Denmark)). Bound

Table 1. - Characteristics of patients with chronic eosinophilic pneumonia $(n=12)$

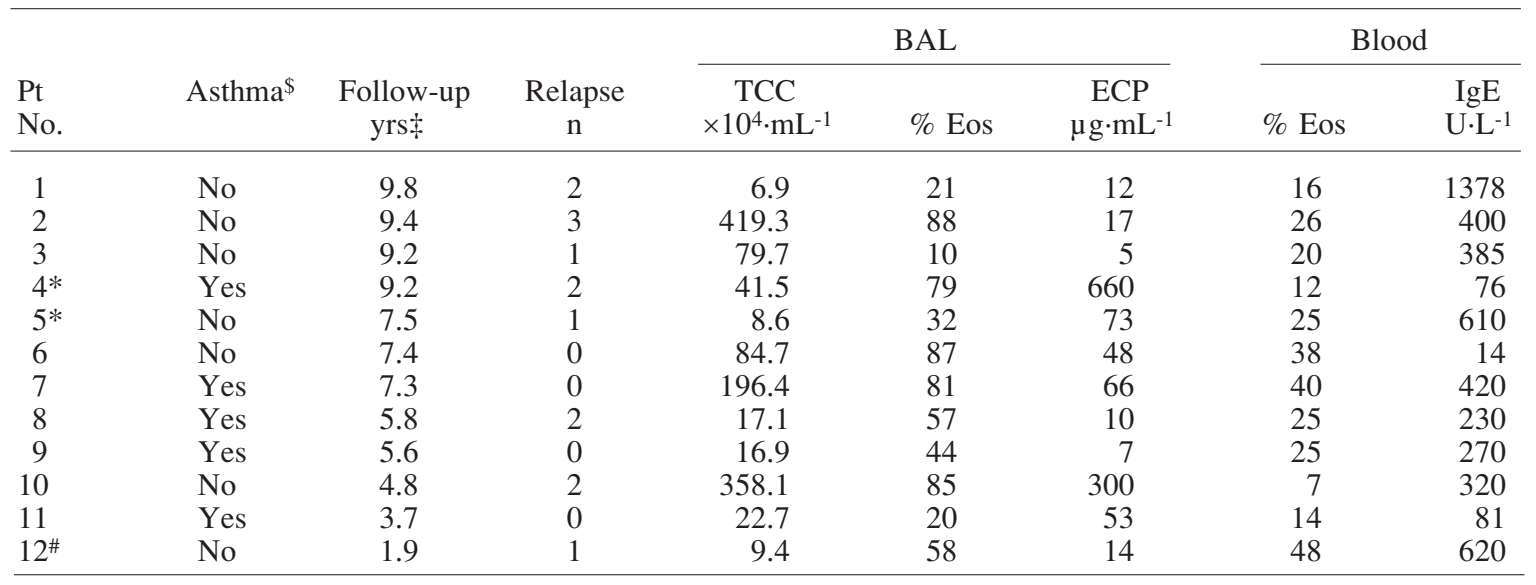

\$: asthma means pre-existing asthma; *: open lung biopsy; \#: crista biopsy, skin biopsy, mediastinoscopy; $\$$ : followup from the time BAL was performed; BAL: bronchoalveolar lavage; Pt: patient; TCC: total cell count; Eos: eosinophils; ECP: eosinophil cationic protein; IgE: immunoglobulin E. 
Igs from BAL fluid were detected by using a horseradish peroxidase (HRP)-labelled rabbit anti-human-Ig antiserum (with anti-IgA, -IgG, -IgM, -kappa, -lambda reactivity), and a chromogenic substrate orthophenyl diamine (OPD; Baker, Chemicals BV, Deventer, The Netherlands). Immunoglobulin concentrations in BAL fluid were expressed in $\mathrm{mg} \cdot \mathrm{L}^{-1}$ using a commercial human standard serum as a reference (H00-03; CLB, Amsterdam, The Netherlands).

\section{ECP}

ECP levels in BAL fluid were measured using a radioimmunoassay (Pharmacia Diagnostics, Uppsala, Sweden) in unconcentrated BAL fluid. The detection range is 2.0$400 \mu \mathrm{g} \cdot \mathrm{L}^{-1}$. The cross-reactivity with eosinophil-derived neurotoxin $(\mathrm{EDN})$ is $<0.06 \%$ and with $\mathrm{EPO}$ is $<0.04 \%$. All assays were performed in duplicate. Samples containing $400 \mu \mathrm{g} \cdot \mathrm{L}^{-1} \mathrm{ECP}$ were diluted and retested.

\section{Statistical analysis}

The Spearman's rank test was used in order to test for a monotonic relationship between ECP levels in BAL fluid and the relative and absolute numbers of eosinophils. The same test was used to test for a relationship between immunoglobulin levels and ECP levels in BAL fluid from patients with CEP. The Mann-Whitney Utest was used to evaluate differences between patients with CEP and healthy controls concerning ECP levels, immunoglobulin levels and cellular analysis of BAL fluid samples. The same test was used to determine whether there would be differences in the ECP levels in BAL fluid from CEP patients with or without certain clinical parameters.

\section{Results}

The results of the BAL fluid analysis concerning the recovery rate, total cell count and the BAL cell profile from the patients with CEP and the control subjects (Control Group A) are presented in table 2. The recovery rate was significantly lower in the patients with CEP compared to the controls, whereas the total cell count and the total cells $\cdot \mathrm{mL}^{-1}$ BAL fluid were significantly increased in the patients with CEP. In addition to the percentage of eosinophils, the percentage of mast cells was significantly elevated in BAL fluid from patients with CEP compared to controls. Moreover, plasma cells were demonstrated in BAL fluid from four patients. The absolute numbers of eosinophils, mast cells, and polymorphonuclear neutrophils (PMNs) were significantly increased in the patients with CEP compared to control subjects, whereas the absolute numbers of alveolar macrophages and lymphocytes did not significantly differ from the controls (data not shown).

ECP levels in BAL fluid from patients with CEP $(n=12)$ were significantly elevated compared to ECP levels in BAL fluid from the 10 control subjects (Control Group $\mathrm{B} ; \mathrm{p}<0.001)$. The mean ECP level in BAL fluid from patients with CEP was $90.0 \mu \mathrm{g} \cdot \mathrm{L}^{-1}$ (SEM $48.8 \mu \mathrm{g} \cdot \mathrm{L}^{-1}$, range 5-660 $\mu \mathrm{g} \cdot \mathrm{L}^{-1}$, median $\left.32.5 \mu \mathrm{g} \cdot \mathrm{L}^{-1}\right)$; whereas, the ECP level was detectable in only one BAL fluid sample from the control subjects, $2.8 \mu \mathrm{g} \cdot \mathrm{L}^{-1}$ (fig. 1). The ratio of ECP levels to albumin in BAL fluid from patients with CEP (mean $1.2 \times 10^{-4}$ ) were also significantly increased compared to the controls (mean $<0.4 \times 10^{-4}$; $\mathrm{p}<0.05$ ). No significant relationship was demonstrated between the percentage of eosinophils and the ECP levels in BAL fluid from patients with CEP; neither was a significant relationship established between the absolute number of eosinophils and ECP levels in BAL fluid. No significant differences were observed between the ECP levels in BAL fluid from patients with CEP, with or without pre-existing asthma; neither was a significant difference demonstrated in the percentage of eosinophils or the absolute number of eosinophils in BAL fluid (data not shown).

The absolute $\operatorname{IgM}, \operatorname{IgG}$ and $\operatorname{Ig}$ A levels and the ratio of the immunoglobulins to albumin in BAL fluid from patients with CEP were significantly elevated compared

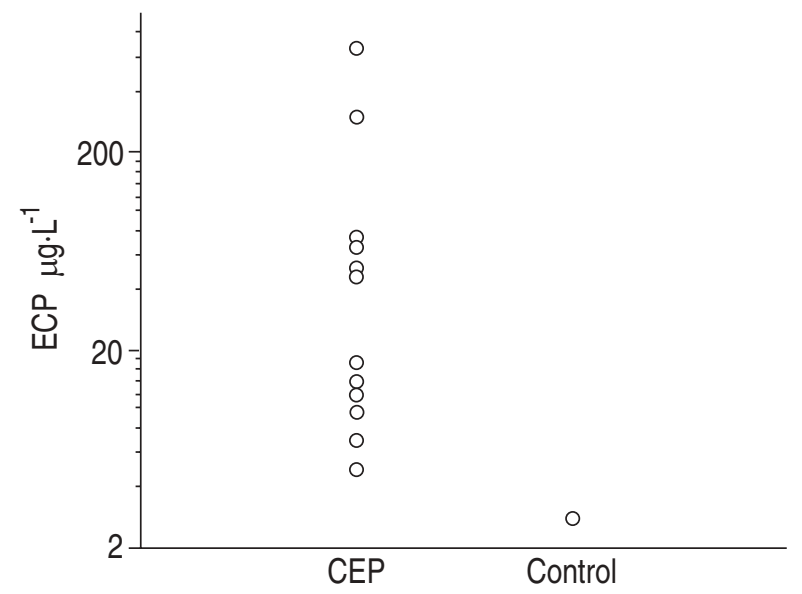

Fig. 1. - Eosinophil cationic protein (ECP) levels in bronchoalveolar lavage fluid obtained from patients with chronic eosinophilic pneumonia (CEP; $\mathrm{n}=12$ ) and Control Group B subjects (controls; $\mathrm{n}=10$ ). The ECP level was detectable in only one of the control subjects.

Table 2. - Total and differential cell count of bronchoalveolar lavage fluid obtained from patients with CEP $(n=12)$ and Control Group A ( $n=15)$, expressed as percentage of the total cell count

\begin{tabular}{|c|c|c|c|c|c|c|c|c|}
\hline & $\begin{array}{c}\text { Yield } \\
\%\end{array}$ & $\begin{array}{c}\text { TCC } \\
\times 10^{4} \cdot \mathrm{mL}^{-1}\end{array}$ & $\underset{\%}{\mathrm{AM}}$ & $\underset{\%}{\mathrm{Lym}}$ & $\underset{\%}{\mathrm{PMN}}$ & $\begin{array}{c}\text { Eos } \\
\%\end{array}$ & $\begin{array}{c}\mathrm{MC} \\
\%\end{array}$ & $\begin{array}{l}\mathrm{PC} \\
\%\end{array}$ \\
\hline CEP & $39 \pm 5^{*}$ & $105.2 \pm 41.5^{\#}$ & $31.5 \pm 8.2 *$ & $8.2 \pm 2.9$ & $3.4 \pm 1.4$ & $55.1 \pm 8.4^{*}$ & $1.65 \pm 0.43^{*}$ & $0.18 \pm 0.09 \#$ \\
\hline Group A & $65 \pm 2$ & $10.1 \pm 1.3$ & $87.0 \pm 1.4$ & $11.0 \pm 1.4$ & $1.6 \pm 1.4$ & $0.3 \pm 0.1$ & $0.07 \pm 0.03$ & $0.00 \pm 0.00$ \\
\hline
\end{tabular}

Data are expressed as mean \pm SEM. CEP: chronic eosinophilic pneumonia; TCC: total cell count; AM: alveolar macrophages; Lym: lymphocytes; PMN: polymorphonuclear neutrophils; Eos: eosinophils; MC: mast cells; PC: plasma cells. *: p<0.001 (MannWhitney U-test), CEP versus Group A; \#: p<0.03 (Mann-Whitney U-test), CEP versus Group A. 
Table 3. - Albumin and immunoglobulin levels in bronchoalveolar lavage fluid of patients with chronic eosinophilic pneumonia $(n=8)$ and Control Group $A(n=10)$

\begin{tabular}{|c|c|c|c|c|c|c|c|}
\hline & $\begin{array}{c}\text { Albumin } \\
\mathrm{mg} \cdot \mathrm{L}^{-1}\end{array}$ & $\begin{array}{l}\operatorname{IgM} \\
\mathrm{mg} \cdot \mathrm{L}^{-1}\end{array}$ & $\begin{array}{c}\mathrm{IgG} \\
\mathrm{mg} \cdot \mathrm{L}^{-1}\end{array}$ & $\begin{array}{l}\operatorname{IgA} \\
\mathrm{mg} \cdot \mathrm{L}^{-1}\end{array}$ & $\operatorname{IgM} /$ albumin & IgG/albumin & IgA/albumin \\
\hline CEP & $930.5 \pm 582.3^{\#}$ & $66.8 \pm 45.7 *$ & $447.1 \pm 224.2 *$ & $74.2 \pm 44.6^{\#}$ & $0.043 \pm 0.11^{\#}$ & $0.52 \pm 0.07 *$ & $0.096 \pm 0.019^{+}$ \\
\hline Group A & $60.5 \pm 6.6$ & $0.4 \pm 0.1$ & $6.6 \pm 1.5$ & $2.4 \pm 0.5$ & $0.006 \pm 0.001$ & $0.11 \pm 0.02$ & $0.47 \pm 0.013$ \\
\hline
\end{tabular}

Values are expressed as mean \pm SEM. CEP: chronic eosinophilic pneumonia; Ig: immunoglobulin. *: p $<0.001$; \#: p<0.002; ${ }^{+}$: p $<0.04$, Mann-Whitney U-test, CEP versus Group A.

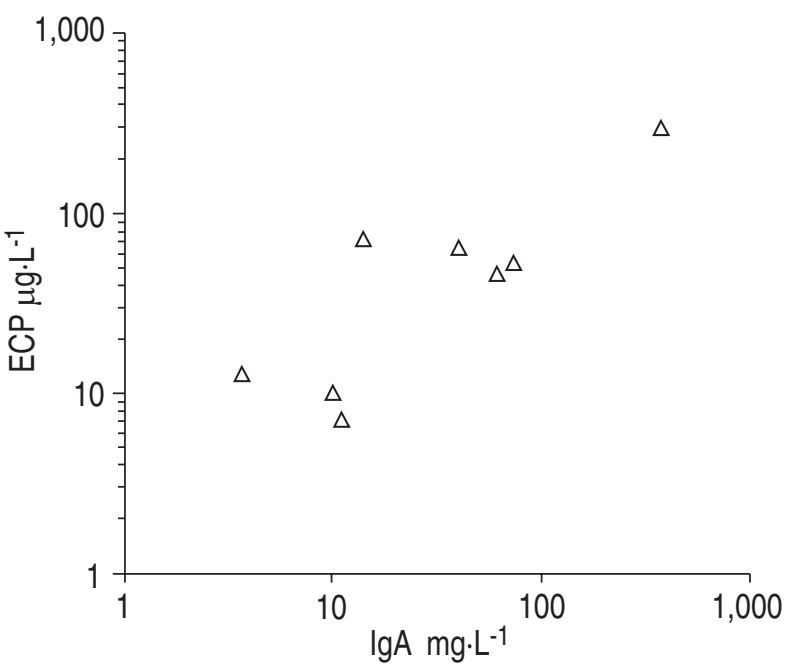

Fig. 2. - Relationship between immunoglobulin A (IgA) levels and eosinophil cationic protein (ECP) levels in bronchoalveolar lavage fluid from patients with chronic eosinophilic pneumonia tested by the Spearman's rank test $(\mathrm{r}=0.72 ; \mathrm{p}=0.043)$.

to the control subjects (Group A) (table 3). A significant relationship was demonstrated between the $\operatorname{IgA}$ and ECP levels in BAL fluid of patients with CEP (fig. 2) $(\mathrm{r}=0.72 ; \mathrm{p}=0.043)$. No such relationship was demonstrated for either IgG or IgM levels.

\section{Discussion}

In this study concerning CEP, we were mainly interested in the number of eosinophils, their state of activation and the immunoglobulin levels in relation to the degranulation of eosinophils. BAL was used as a tool to obtain a representative sample of the epithelial lining fluid (ELF) containing solutes and a population of cells which are important to the pathophysiological process of the disease under investigation. In this study, ECP levels in BAL fluid samples obtained from 12 patients with CEP were found to be significantly elevated compared to ECP levels in BAL fluid obtained from healthy control subjects. With the lavage procedure, ELF is diluted in BAL fluid to a variable degree [20]. So far, no definite method has been established, to correct for the dilutional effect of BAL. The concentration of albumin in BAL fluid is often used as a reference marker, but in interstitial lung diseases in particular this method is questionable, since the influx of albumin from the circulation is enhanced due to the increased permeability of the alveolar membrane [21]. However, the ratios of ECP to albumin in BAL fluid from patients with CEP were also significantly increased compared to the controls. The presence of such high concentrations of ECP in the BAL fluid of patients with CEP indicates that eosinophils in the lung parenchyma and in the interstitium were present in an activated state and had degranulated. This was also demonstrated by histopathological studies, in which degraded eosinophils and extracellular granules, ECP and MBP were observed [7].

Our results confirm data obtained by JANIN et al. [16], who determined ECP levels in BAL fluid from only three patients with CEP, and data concerning six patients with CEP recently described by SHIJUBO et al. [17]. However, in contrast to the latter study, we did not demonstrate a relationship between the absolute number of eosinophils and ECP levels in BAL fluid obtained from patients with CEP (using the Spearman's rank test). This may indicate that the percentage of activated eosinophils in the pulmonary compartment may differ between patients. A lack of association between the eosinophil content and the ECP level in BAL fluid was recently described, concerning a patient with CEP progressing to lung fibrosis [22]. In the present study, no significant differences were found in clinical symptoms, including the number of relapses and the overall prognosis, between patients with a higher percentage or a lower percentage of activated eosinophils in BAL fluid.

As reported by others previously, ECP levels in BAL fluid from patients suffering from asthma were found to be elevated $[23,24]$. The ECP levels in BAL fluid from patients with CEP with pre-existing asthma tended to be higher than those of patients without pre-existing asthma, but the differences did not reach significance. It is, however, possible that in a study including more patients such a relationship might be demonstrated.

The stimuli or the processes which lead to the degranulation of eosinophils in CEP remain to be elucidated. In vitro studies have demonstrated degranulation of eosinophils in response to aggregated immunoglobulins and immune complexes. Eosinophils express receptor molecules for $\operatorname{IgA}$, IgG, and $\operatorname{IgE}$ on the plasma $[25,26]$. Aggregated $\operatorname{IgG}$ and $\operatorname{IgA}$ stimulate release of ECP by eosinophils [10], whereas, in response to IgE only EPO is released [11]. In this study, the $\operatorname{IgG}, \operatorname{IgA}$, and $\operatorname{IgM}$ levels in BAL fluid from patients with CEP were significantly increased compared to the immunoglobulin levels in the lavage fluid of the control subjects. Additionally, the IgG/albumin, IgA/albumin, and IgM/albumin ratios in BAL fluid from patients with CEP were also significantly elevated compared to controls. This is, in part, in contrast to data described by Pesci et al. [27], who demonstrated significantly increased IgG/ albumin ratios in BAL fluid of six CEP patients but 
normal $\operatorname{IgA}$ /albumin ratios (IgM levels were not studied by these investigators).

The increased immunoglobulin levels in BAL fluid demonstrated in the present study are, to a major extent, caused by leakage from the blood vessels through the respiratory membrane into the pulmonary compartment, due to the inflammatory process. However, local production of IgA in CEP may be enhanced, since the IgA/ albumin ratio in BAL fluid is significantly higher than the $\operatorname{IgA}$ /albumin ratio in serum $(\mathrm{p}<0.04)$. On the other hand, relatively more leakage across the alveolar membrane has been described for proteins with higher molecular weight [28]. However, the presence of plasma cells in the BAL fluid from four patients suggests that, at least in some patients, local production of immunoglobulins will be increased.

Measurement of secretory IgA could be a method to confirm the enhanced local production of IgA in CEP. The significant relationship demonstrated in this study between IgA levels and ECP levels in BAL fluid from patients with CEP may suggest that (complexed) IgA induces degranulation of eosinophils and secretion of ECP in these patients. However, it cannot be excluded that the increase of the IgA levels, due to enhanced permeability of the alveolar epithelial barrier, is to a certain extent caused by the toxic effects of ECP. On the other hand, a significant relationship was demonstrated only between IgA and ECP levels and not between IgG and ECP levels. Furthermore, in vitro studies demonstrated that aggregated IgA (especially secretory IgA) provided a more potent signal for eosinophil degranulation than aggregated $\mathrm{IgG}$.

In conclusion, this study demonstrated that eosinophil cationic protein and immunoglobulin levels are significantly increased in bronchoalveolar lavage fluid from patients with chronic eosinophilic pneumonia. The relationship found between the immunoglobulin $\mathrm{A}$ and eosinophil cationic protein levels in bronchoalveolar lavage fluid from patients with chronic eosinophilic pneumonia is compatible with the hypothesis that immunoglobulin $\mathrm{A}$ is involved in the degranulation of eosinophils. Future prospective (experimental) studies will be needed to investigate a possible underlying mechanism.

Acknowledgements: The authors gratefully acknowledge the technical assistance of E. Tuenter and E.J van Scheijen.

\section{References}

1. Jederlinic PJ, Sicilian L, Gaensler EA. Chronic eosinophilic pneumonia: a report of 19 cases and a review of the literature. Medicine (Baltimore) 1988; 67: 154-162.

2. Carrington CB. Eosinophilic reactions in the lung. $N$ Engl J Med 1969; 280: 787-798.

3. Pearson DL, Rosenow EC. Chronic eosinophilic pneumonia (Carrington's): a follow-up study. Mayo Clin Proc 1978; 53: 73-78.

4. Hayakawa H, Sato A, Toyoshima M, Imokawa S, Taniguchi M. A clinical study of idiopathic eosinophilic pneumonia. Chest 1994; 105: 1462-1466.

5. Naughton M, Fahy J, Fitz Gerald MX. Chronic eosinophilic pneumonia: a long-term follow-up of 12 patients. Chest 1993; 103: 162-165.
6. Fox B, Seed WA. Chronic eosinophilic pneumonia. Thorax 1980; 35: 570-580.

7. Gonzalez EB, Swedo JL, Rajaraman S, Daniels JC, Grant JA. Ultrastructural and immunohistochemical evidence for release of eosinophilic granules in vivo: cytotoxic potential in chronic eosinophilic pneumonia. J Allergy Clin Immunol 1986; 79: 755-762.

8. Ayars GH, Altman LC, Gleich GJ, Loegering DA, Baker CB. Eosinophil- and eosinophil granule-mediated pneumocyte injury. J Allergy Clin Immunol 1985; 76: 595-604.

9. Davis WB, Fells GA, Sun XH, Gadek JE, Venet A, Crystal RG. Eosinophil-mediated injury to lung parenchymal cells and interstitial matrix: a possible role for eosinophils in chronic inflammatory disorders of the lower respiratory tract. J Clin Invest 1984; 74: 269278.

10. Abu Ghazaleh RI, Fujisawa T, Mestecky J, Kyle RA, Gleich GJ. IgA-induced eosinophil degranulation. $J$ Immunol 1989; 142: 2393-2400.

11. Tomassini M, Tsicopoulos A, Tai PC, et al. Release of granule proteins by eosinophils from allergic and nonallergic patients with eosinophilia on immunoglobulindependent activation. J Allergy Clin Immunol 1991; 88: 365-375.

12. Tai PC, Capron M, Bakes DM, Barkans J, Spry CJ. Monoclonal antibodies to human eosinophil plasma membrane antigens enhance the secretion of eosinophil cationic protein. Clin Exp Immunol 1986; 63: 728-737.

13. Gustafsson R, Fredens K, Nettelbladt O, Hallgren R. Eosinophil activation in systemic sclerosis. Arthritis Rheum 1991; 34: 414-422.

14. Hallgren R, Bjermer L, Lundgren R, Venge P. The eosinophil component of the alveolitis in idiopathic pulmonary fibrosis: signs of eosinophil activation in the lung are related to impaired lung function. Am Rev Respir Dis 1989; 139: 373-377.

15. Hallgren R, Samuelsson T, Venge P, Modig J. Eosinophil activation in the lung is related to lung damage in adult respiratory distress syndrome. Am Rev Respir Dis 1987; 135: 639-642.

16. Janin A, Torpier G, Courtin P, et al. Segregation of eosinophil proteins in alveolar macrophage compartments in chronic eosinophilic pneumonia. Thorax 1993; 48: $57-62$.

17. Shijubo N, Shigehara K, Hirasawa M, Inuzuka M, Abe S. Eosinophilic cationic protein in chronic eosinophilic pneumonia and eosinophilic granuloma. Chest 1994; 106: $1481-1486$

18. American Thoracic Society. Medical section of the American Lung Association: standards for the diagnosis and care of patients with chronic obstructive pulmonary disease (COPD) and asthma. Am Rev Respir Dis 1987; 137: 225-244.

19. Drent M, Mulder PG, Wagenaar SjSc, Hoogsteden HC, van Velzen Blad H, van den Bosch JM. Differences in BAL fluid variables in interstitial lung diseases evaluated by discriminant analysis. Eur Respir J 1993; 6: 803-810.

20. Kelly CA, Fenwick JD, Corris PA, Fleetwood A, Hendrick DJ, Walters EH. Fluid dynamics during bronchoalveolar lavage. Am Rev Respir Dis 1988; 138: 81-84.

21. Jones KP, Edwards JH, Reynolds SP, Peters TJ, Davies BH. A comparison of albumin and urea as reference markers in bronchoalveolar lavage fluid from patients with interstitial lung disease. Eur Respir J 1989; 3: 152-156. 
22. Yoshida K, Shijubo N, Koba H, et al. Chronic eosinophilic pneumonia progressing to lung fibrosis. Eur Respir J 1994; 7: 1541-1544.

23. Venge P, Henriksen J, Dahl R. Eosinophils in exerciseinduced asthma. J Allergy Clin Immunol 1991; 88: 699-704.

24. Rak S, Bjornson A, Hakanson L, Sorenson S, Venge P. The effect of immunotherapy on eosinophil accumulation and production of eosinophil chemotactic activity in the lung of subjects with asthma during the natural pollen season. Eur Respir J 1991; 88: 878-888.

25. Kroegel C, Virchow JCJ, Luttmann W, Walker C, Warner
JA. Pulmonary immune cells in health and disease: the eosinophil leucocyte. Part I. Eur Respir J 1994; 7: 519-543.

26. Kerr MA. The structure and function of human IgA. Review article. Biochem J 1990; 271: 285-296.

27. Pesci A, Bertorelli G, Manganelli P, et al. Bronchoalveolar lavage in chronic eosinophilic pneumonia: analysis of six cases in comparison with other interstitial lung diseases. Respiration 1988; 54 (Suppl. 1): 16-22.

28. Out TA, van de Graaf EA, Jansen HM. Permeability or local production of immunoglobulins and other inflammatory proteins in asthma. Eur Respir J 1991; 13 (Suppl.): $148 \mathrm{~s}-155 \mathrm{~s}$. 